\title{
The reverse Magnus effect in golf balls
}

\author{
Bin Lyu ${ }^{1} \cdot$ Jeffery Kensrud ${ }^{1} \cdot$ Lloyd Smith $^{1}$
}

Published online: 6 February 2020

(c) The Author(s) 2020

\begin{abstract}
The following considers the lift and drag response of three commercially available golf balls. The balls were projected with spin through still air in a laboratory setting to investigate a reverse Magnus effect, where balls move in the opposite direction of the expected lift force. The drag and lift coefficients were found by measuring ball position and speed at three points along its trajectory. Three ball types, with different dimple patterns, exhibited reverse Magnus behavior between $5 \times 10^{4}<\operatorname{Re}<7 \times 10^{4}$ and $750<\omega<2250 \mathrm{rpm}$. The golf ball with circular dimples had the least severe reverse Magnus effect, $C_{\mathrm{L}}=-0.1$, while the ball with hexagonal dimples had the greatest, $C_{\mathrm{L}}=-0.15$. The magnitude of the reverse Magnus effect was related to the drag crisis of each ball model. As the slope of the drag crisis became steeper, the magnitude of the reverse Magnus effect increased.
\end{abstract}

Keywords Aerodynamics · Reverse Magnus · Golf balls · Lift · Drag

\section{Introduction}

The aerodynamics of a golf ball is of interest [1] as it is the fastest sport's ball with the longest carry distance [2]. Small changes in aerodynamic properties can influence the flight behavior of the ball and the result of the game.

Drag, $F_{\mathrm{D}}$, is the force acting in the direction opposing the ball's flight path. Lift, $F_{\mathrm{L}}$, is the force acting perpendicular to the ball's trajectory. The drag and lift coefficients, $C_{\mathrm{D}}$ and $C_{\mathrm{L}}$, are defined by

$C_{\mathrm{D}}=\frac{2 F_{\mathrm{d}}}{\rho A V^{2}}, \quad C_{\mathrm{L}}=\frac{2 F_{\mathrm{L}}}{\rho A V^{2}}$

where $\rho$ is the density of air, $A$ is the cross-sectional area of the ball, and $V$ is the speed of the ball. The cause of the movement of the ball is related to flow separation from its surface. The speed of the ball can be expressed in a dimensionless quantity known as the Reynolds number (Re), defined by

$\operatorname{Re}=\frac{V D}{v}$

Lloyd Smith

lvsmith@wsu.edu

1 Washington State University, Pullman, USA where $D$ is diameter of the ball, and $\nu$ is kinematic viscosity of air.

A drag crisis is observed when the ball's coefficient of drag becomes unstable and decreases quickly with increasing ball speed. This phenomenon is a result of a transition from a laminar (Fig. 1a) to a turbulent (Fig. 1b) boundary layer [3]. Laminar boundary layers are more prone to separation than turbulent boundary layers because they have less momentum near the surface. As the ball speed increases, turbulence forms in the boundary layer, delaying the flow separation point, and decreasing the size of the wake. In play, golf balls usually travel at speeds that are near the end or after the drag crisis (launch speed between 20 and $94 \mathrm{~m} / \mathrm{s}$ ) [4].

For balls with spin, it is convenient to describe the ratio of the angular velocity to the linear velocity by the nondimensional spin factor [5], $S$, defined by

$S=\frac{\omega r}{V}$

where $\omega$ is the angular velocity, and $r$ is the ball radius.

With backspin, the top of the ball is moving with the air flow, while the bottom of the ball is moving against the air flow. The relative air to ball surface speed on the top and bottom of the ball is, therefore, lower and higher than the ball speed, respectively. Backspin causes flow on the top side to separate further from the stagnation point, while flow on the bottom side separates closer to the stagnation 
Fig. 1 Flow from left to right over a golf ball at different Re and $S$. Spin rate for each one: a laminar boundary layer: $\operatorname{Re}<5 \times 10^{4}, S=0$. b Turbulence boundary layer: $\operatorname{Re}>7.5 \times 10^{4}, S=0$. c Magnus effect: $\operatorname{Re}=6.5 \times 10^{4}, S=0.24$ (2500 rpm). d Reverse Magnus effect: $\operatorname{Re}=6.5 \times 10^{4}, S=0.12$ (1250 rpm) [9]. The red dots indicate stagnation point, while the green dots indicate separation points. The red arrows indicate the direction of lift force

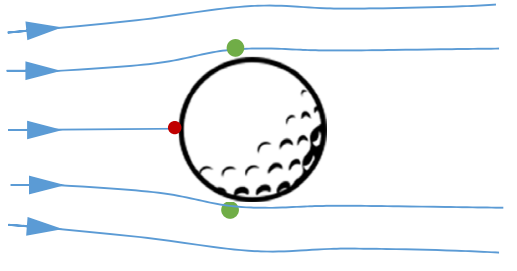

a

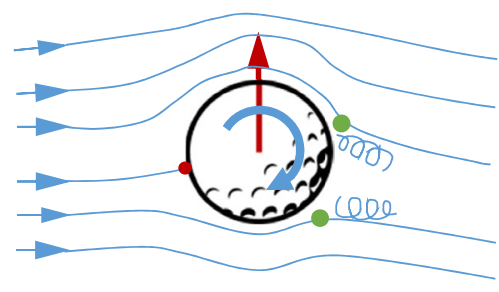

C

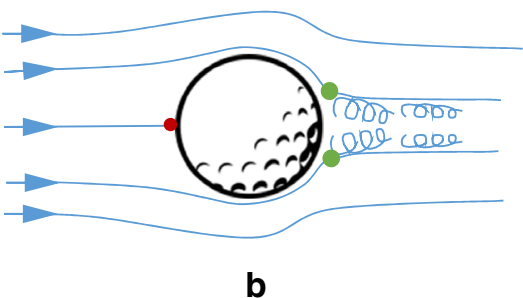

b

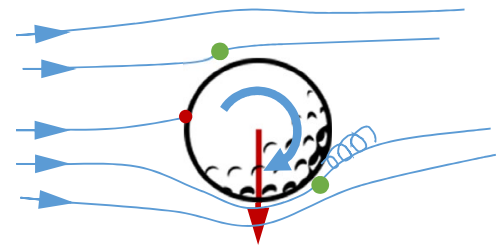

d point (Fig. 1c). The rotated stagnation points (relative to the ball center) cause a vertical pressure gradient, producing an upward force and the Magnus effect [6, 7].

For specific conditions, one study has shown a negative lift force on a sphere with backspin, referred to as the reverse Magnus effect [8]. When the ball is traveling near the speed where the boundary layer becomes turbulent, backspin can cause the top side of the ball to have laminar flow, while the bottom boundary layer is turbulent, as shown in Fig. 1d [9]. The laminar flow on the top surface moves the separation point forward, changing the direction of the Magnus effect downward, producing the reverse Magnus effect.

Only a few studies have observed the reverse Magnus effect due to the small range of speed and spin rate where it occurs. Using the wind tunnel, Bearman and Harvey found the reverse Magnus effect on scaled model golf balls at low speed $(13.7 \mathrm{~m} / \mathrm{s})$ and low spin rate $(<1500 \mathrm{rpm})$ [9]. Reverse Magnus was found numerically and experimentally on smooth balls [10-12]. Bush found that the reverse Magnus effect on smooth spheres occurred in the drag crisis region [13]. Kim et al. [8] correlated the location of flow separation as a function of the speed and the spin rate for reverse Magnus. Oggiano compared soccer balls experimentally and numerically and identified a reverse Magnus effect on one of four ball models [14]. Barber et al. [15] showed that the soccer ball lift is more sensitive to ball design than drag, which may explain why Oggiano only saw reverse Magnus with one ball model. While others have shown the reverse Magnus effect occurs at speeds in the drag crisis, no study has related the magnitude of the reverse Magnus effect to the drag crisis.

While wind tunnels are often used to study aerodynamic behavior of golf balls, disparities between laboratory and play conditions remain. Wind tunnels are good at controlling air speed and ball orientation, while challenges exist in supporting the ball and adding spin [9]. Wind tunnels have difficulty testing golf balls at sport-like speeds and often use scaled ball models to achieve Reynolds numbers representative of play. Wind tunnels must also correct for blockage effects using empirical factors, which can be challenging to identify [16]. The authors have found no published study measuring the reverse Magnus effect of golf balls, rather than scaled ball models in wind tunnels. Given the sensitivity of golf ball drag [17], small differences between scaled and actual golf balls can be important.

Projecting balls through still air avoids many of the challenges encountered with balls in wind tunnels. Recent advances in high-speed videography [18], light gates [19], and radar tracking [20] have improved the accuracy of inflight trajectory measurements in and outside the laboratory [21-23]. The aim of this study was to consider the reverse Magnus effect of golf balls traveling through still air. The relationship between the drag crisis and the reverse Magnus effect was quantified. The aerodynamic characteristics and the flow conditions that induce the reverse Magnus effect for commercially available golf balls were also identified.

\section{Methods}

This study considered three golf balls with different dimple patterns, as described in Table 1.

Balls were projected in laboratory still air with a bespoke, non-wheeled, pitching machine (see Fig. 2a) up to $\operatorname{Re}=25 \times 10^{4}$ and $\omega=3000 \mathrm{rpm}$ without damaging or disrupting the surface of the ball. The machine used a pneumatic linear accelerator to achieve the target speed. A flexible tip was coupled to the shaft and used to impart rotation to the ball, where high- and low-friction materials on opposite sides generated torque about the center 
Table 1 Description of golf balls used in this study
Fig. 2 a Setup on the pitching machine to generate backspin. b Arrangement of pitching machine, speed sensors, and camera to measure ball speed, rotation, and location. Gates aligned vertically measured ball speed, while angled gates measured position

\begin{tabular}{llll}
\hline Brand & Titleist & Callaway & Bridgestone \\
\hline Model & Pro V1 & ChromeSoft & B330-RX \\
Code & V1 & CA & BS \\
Cover & Urethane elastomer & Thermoplastic urethane & Urethane \\
Construction & 3-Piece & 4-Piece & 3-Piece \\
Dimple shape & Circular & Hexagonal & Dual \\
Dimple number & 352 & 332 & 330 \\
Dimple image & & & \\
& & & \\
& & & \\
\hline
\end{tabular}
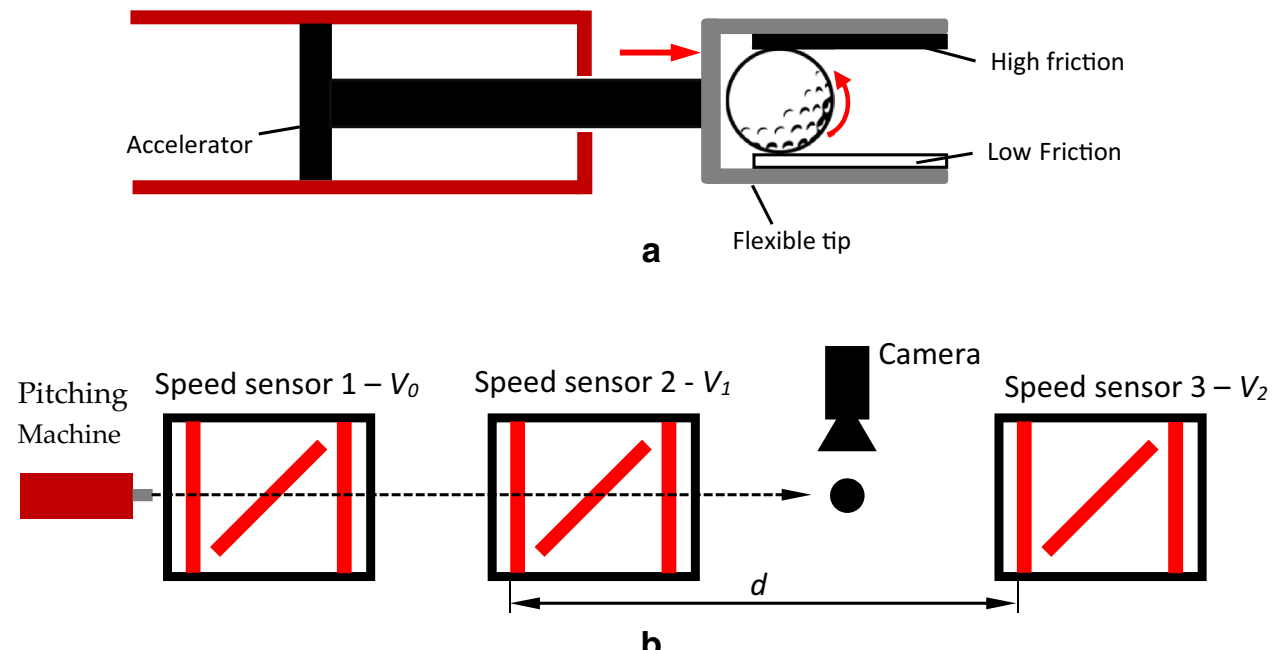

of the ball [24]. Spin rate was controlled by changing the distance the ball rolled in the flexible tip.

Ball speed and location were measured at three locations along the flight path, as shown in Fig. 2b. Speed was measured from speed sensors; each composed of a pair of light gates $0.41 \mathrm{~m}$ apart (Ibeam, ADC, Romeoville, IL, USA). The distance, $d$, between the second and third speed sensors was $3.81 \mathrm{~m}$, while the heights were within $0.025 \mathrm{~m}$ of each other. Sensors were placed to maximize the distance between them while minimizing the change in trajectory angle. (The change in the vertical position of the ball was $0.1 \mathrm{~m}$ or less.) An angled light gate was used to measure the ball's vertical location at each speed sensor to obtain the lift force. A high-speed video camera (Phantom V711) recorded each shot at 2000 fps to verify orientation and spin rate (within $\pm 15 \mathrm{rpm}$ ). The drag force, $F_{\mathrm{D}}$, was found from
$F_{\mathrm{D}}=m \frac{V_{2}^{2}-V_{1}^{2}}{2 d}$

where $V_{2}$ and $V_{1}$ were the speeds at the second and third sensors, respectively, and $m$ was the ball mass. The lift force, $F_{\mathrm{L}}$, was found from

$F_{\mathrm{L}}=m\left(\frac{2\left(D_{y}-V_{y} t_{2}\right)}{t_{2}^{2}}-g\right)$

where $D_{y}$ was the vertical change in ball position between the second and third speed sensors, $V_{y}$ was the vertical velocity generated from the launch angle, $t_{1}$ was the time from the first speed sensor to the second speed sensor, $t_{2}$ was the time for the ball to travel from the second speed sensor to the third speed sensor, and $g$ was gravity. Although the balls were projected horizontally, imparting spin tended to result 
in a small unknown vertical speed at the pitching machine. The vertical component of velocity at the second sensor, $V_{y}$, was found from the known speeds and locations at all three sensors. Details of the lift and drag measurements may be found elsewhere [25].

\section{Calibration and uncertainty}

The coefficient of lift and drag was calibrated by testing a setup golf ball daily prior to testing. The speed and spin rate of the calibration shots were the same as those used for the tested golf balls. To calibrate lift, the setup golf ball was projected with a vertical spin axis to prevent a Magnus effect from occurring in the vertical direction. The position of the speed sensors was adjusted until the lift coefficient from three shots was $0.00 \pm 0.01$. To ensure test repeatability, a setup golf ball was projected through the system at the start of each 4-h session. The sensor positions were adjusted until its drag coefficient was within \pm 0.02 of its initial value.

The precision of the speed sensor was $0.025 \%$ of the measured speed [25]. Accordingly, the uncertainty of $C_{\mathrm{D}}$ and $C_{\mathrm{L}}$ for a golf ball was 0.005 and 0.0005 , respectively. Other factors could affect the uncertainty of the drag and lift measurement, including error from air properties and position measurement. Random error was analyzed by measuring the variance of 13 balls at eight test speeds. Each ballspeed combination consisted of four repeat shots. The mean standard deviation of the drag and lift coefficient for all 416 tests was 0.02 and 0.01 , respectively, for a golf ball traveling between 30 and $70 \mathrm{~m} / \mathrm{s}$ at $2250 \mathrm{rpm}$.

\section{Results}

$C_{\mathrm{L}}$ and $C_{\mathrm{D}}$ as a function of Re are shown in Fig. 3. The reverse Magnus effect was observed for all three ball models when $\mathrm{Re}=6.5 \times 10^{4}$.

The lift coefficient of V1 is plotted as a function of spin factor in Fig. 4 for $5 \times 10^{4}<\operatorname{Re}<7.5 \times 10^{4}$. When $\operatorname{Re}>7 \times 10^{4}$, the lift coefficient increased steadily with spin rate. When $\operatorname{Re}<7 \times 10^{4}$, the dependence of the lift coefficient on speed was complex. Positive lift decreased with decreasing speed until becoming negative when $\mathrm{Re}=6 \times 10^{4}$ and $0.1<S<0.23$ (based on the trend line). Positive lift reoccurred when $\operatorname{Re} \leq 5 \times 10^{4}$.

The reverse Magnus effect was also found on the other two ball models. Ball lift is presented in Fig. 5 for the

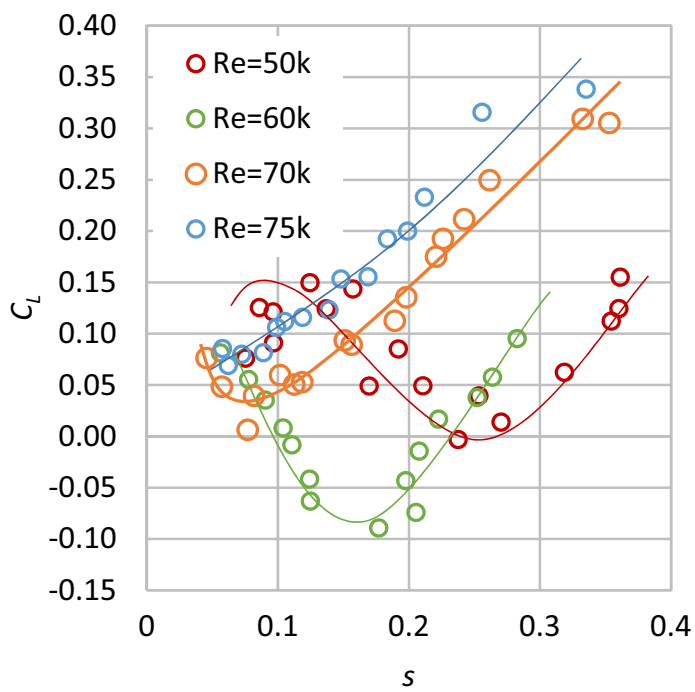

Fig. 4 Lift coefficient of V1 as a function of spin factor. Four representative speeds are presented in the plot
Fig. 3 Drag (left) and lift (right) coefficients as a function of Reynolds number for three golf ball models, $2250 \pm 250 \mathrm{rpm}$ $\left(S=0.21\right.$ when $\left.\operatorname{Re}=6.5 \times 10^{4}\right)$. Each data point is the mean of four shots where the error bars represent one standard deviation
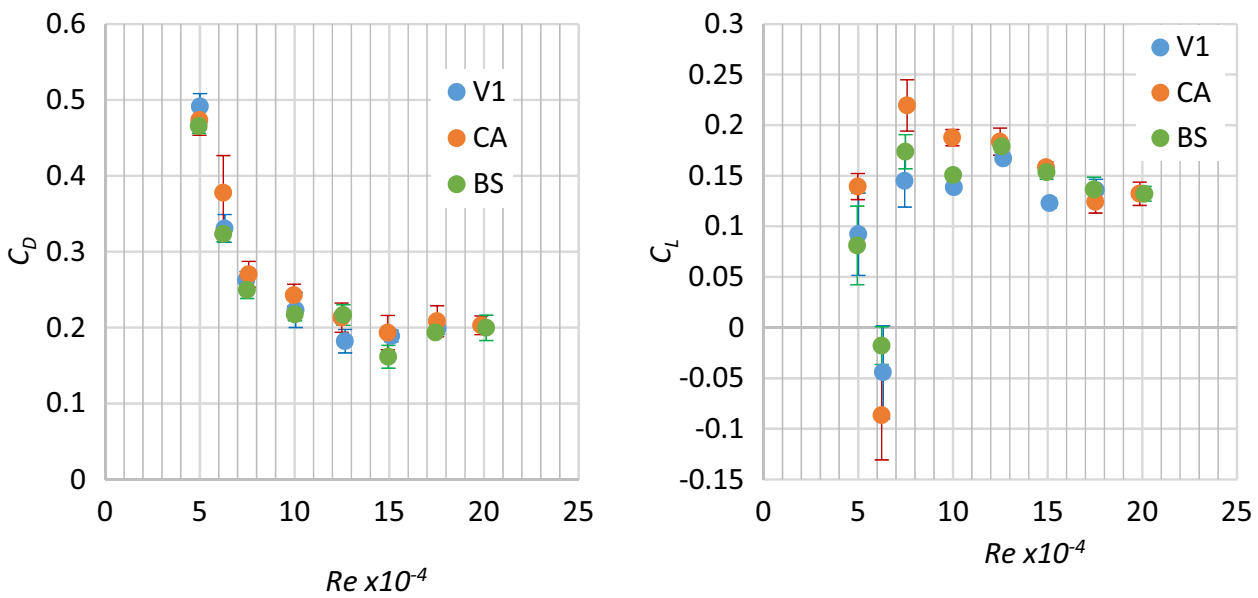


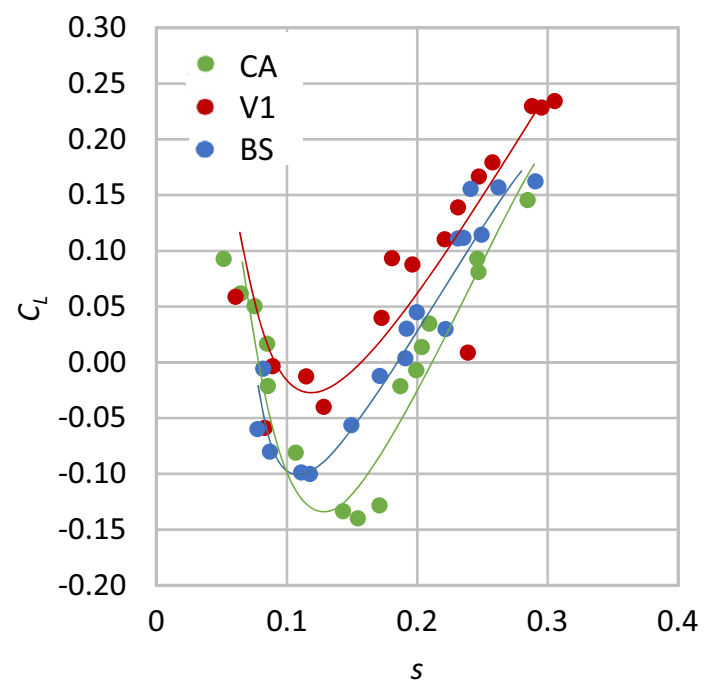

Fig. 5 Lift coefficient of three ball models as a function of spin factor at $\operatorname{Re}=6.5 \times 10^{4}$

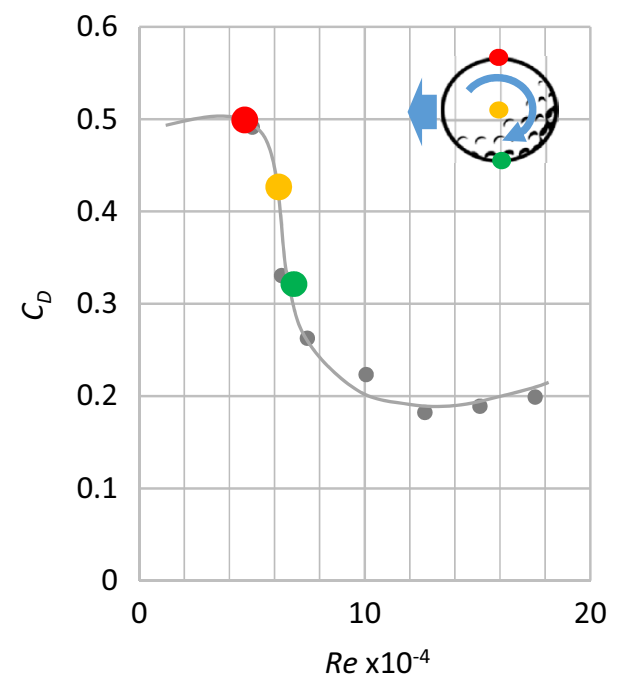

Fig. 6 Drag coefficient of V1 as a function of Reynolds number. The blue arrows on the ball indicate the traveling and spinning directions. The yellow dot on the curve indicates the drag coefficient when $\mathrm{Re}=6 \times 10^{4}$ and $\omega=1500 \mathrm{rpm}$. The red and green dots indicate the local drag coefficient and Reynolds number on the top and bottom surfaces, respectively

three ball models at $\mathrm{Re}=6.5 \times 10^{4}$. CA showed the most severe reverse Magnus effect with the lowest lift coefficient. V1 showed the least severe reverse Magnus effect. $\mathrm{V} 1$ also showed a negative lift coefficient in a narrower range of spin $(0.09<s<0.15)$ than $\mathrm{BS}(0.07<s<0.19)$ and CA $(0.08<s<0.21)$.

\section{Discussion}

The foregoing has shown a consistent reverse Magnus effect of three golf ball models. The reverse Magnus effect has only been observed for balls traveling at speeds in the drag crisis [13]. The reverse Magnus effect is likely dominant in golf balls due to their severe drag crisis, as shown in Fig. 6 for V1 [26]. The drag coefficient for V1 changed from 0.5 to 0.2 when the Reynolds number increased from $5 \times 10^{4}$ to $7.5 \times 10^{4}$. Similar results for other golf balls have been observed elsewhere [9, 17].

The drag coefficient is typically used to describe the air resistance of an object. For the case of a ball with backspin, traveling at speeds in the drag crisis, the flow rate at the top and bottom surfaces can be different. For such cases, it is illustrative to consider a local speed from the relative air ball surface. Thus, the local speed of the bottom surface is $V+r$, while the local speed of the top surface is $V-r$. A ball traveling at $\operatorname{Re}=6 \times 10^{4}$ and $\omega=1500 \mathrm{rpm}$, for instance, would have a local bottom surface $\operatorname{Re}=6.9 \times 10^{4}$ and a local top surface $\operatorname{Re}=5.1 \times 10^{4}$, as illustrated in Fig. 6.

Due to the different boundary layer flows on the top (laminar) and the bottom (turbulence) surfaces of the balls, the reverse Magnus effect is only observed in the drag crisis region, where the flow on the ball surface transitions from laminar to turbulence. This study has demonstrated reverse Magnus for the first time on golf balls (Fig. 4) in still air.

In the following, the local slope of the drag crisis $\left(\partial C_{\mathrm{D}} / \partial \mathrm{Re}\right)_{\mathrm{L}}$ was measured at 10 different $\mathrm{Re}$ over $5 \times 10^{4}<\operatorname{Re}<7.5 \times 10^{4}$ (Fig. $7 \mathrm{a}$ ). Here, $\partial C_{\mathrm{D}}$ and $\partial \mathrm{Re}$ were the change in the local $C_{\mathrm{D}}$ and Re, respectively, at the top and bottom of the ball. The local slope of the drag crisis is compared to the lift coefficient for each ball model in Fig. 7b. A correlation between the $\partial C_{\mathrm{D}} / \partial \mathrm{Re}$ and $C_{\mathrm{L}}$ was observed for all the balls tested $\left(r^{2}=0.87\right)$. The slope of the entire drag crisis $\left(\partial C_{\mathrm{D}} / \partial \mathrm{Re}\right)_{\mathrm{E}}$ was found from a linear fit of the entire drag crisis region. As illustrated in Fig. 1c, the Magnus (or reverse Magnus) effect will increase as the asymmetry of the separation points (at the top and the bottom of the ball) increases. A golf ball with a steeper drag crisis showed a more severe maximum reverse Magnus effect (Fig. 8), since it had a larger asymmetry of the separation points.

Although the reverse Magnus effect is not common in long golf drives (due to the low speed and spin rate where it occurs), it may still occur near the green during short chip shots. Since most sport balls have a turbulent boundary layer and a drag crisis, they should likewise exhibit a reverse Magnus effect. The lift coefficient of a baseball was measured and is presented in Fig. 9 at $\mathrm{Re}=8.4 \times 10^{4}$, 
Fig. 7 a Drag coefficient as a function of Reynolds number in the drag crisis region. $\mathbf{b}$ Lift coefficient as a function of the slope of the drag crisis

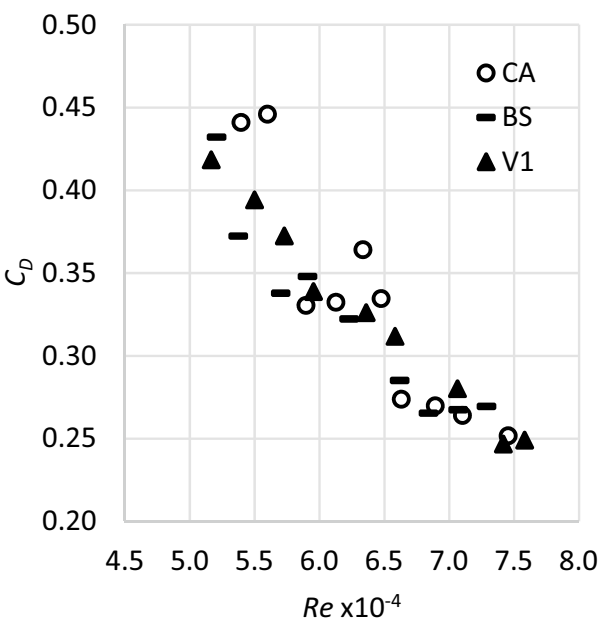

(a)

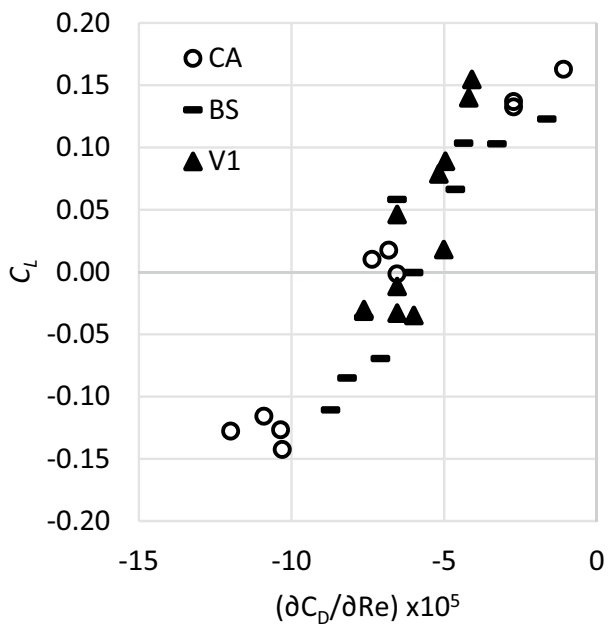

(b)

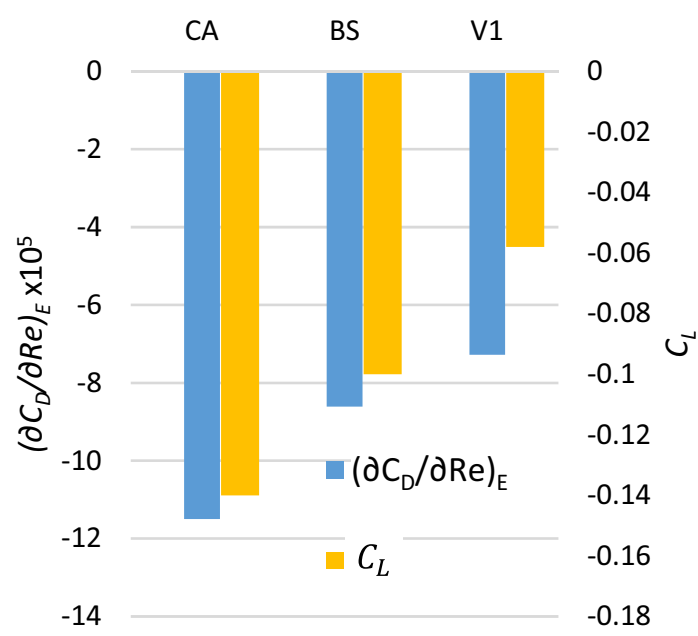

Fig. 8 The comparison of $\partial C_{\mathrm{D}} / \partial \mathrm{Re}$ and the minimum lift coefficient for each ball model

which is in the drag crisis region [25]. The reverse Magnus effect was observed over $0.06<S<0.2$. This condition can occur in play at a high launch angle (greater than $40^{\circ}$ ) as the ball approaches its apex. The findings show that reverse Magnus is not unique to golf, but likely occurs for any sports ball exhibiting a drag crisis, independent of its size.

\section{Summary}

The reverse Magnus effect of three golf ball models has been compared. The lift and drag coefficient of golf balls were measured by projecting them through still air in a laboratory setting. A reverse Magnus effect was observed in the drag crisis region of each ball, as the boundary layer flow

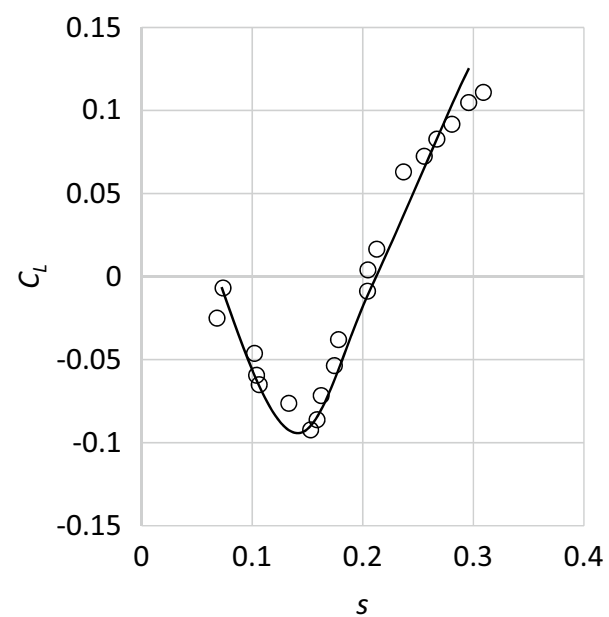

Fig. 9 Lift coefficient of a baseball as a function of spin rate at $\operatorname{Re}=8.4 \times 10^{4}(17 \mathrm{~m} / \mathrm{s})$

transitioned from laminar to turbulent. The magnitude of the reverse Magnus effect was observed to increase with the slope of the drag crisis, and varied with the dimple geometry. The reverse Magnus effect is also expected to occur for other sports balls, evidence of which was presented for a baseball. The reverse Magnus effect occurred at relatively low speeds for golf balls, minimizing its effect on play.

Open Access This article is licensed under a Creative Commons Attribution 4.0 International License, which permits use, sharing, adaptation, distribution and reproduction in any medium or format, as long as you give appropriate credit to the original author(s) and the source, provide a link to the Creative Commons licence, and indicate if changes were made. The images or other third party material in this article are included in the article's Creative Commons licence, unless indicated otherwise in a credit line to the material. If material is not included in the article's Creative Commons licence and your intended use is not permitted by statutory regulation or exceeds the permitted use, you will 
need to obtain permission directly from the copyright holder. To view a copy of this licence, visit http://creativecommons.org/licenses/by/4.0/.

\section{References}

1. Goff JE (2013) A review of recent research into aerodynamics of sport projectiles. Sports Eng 16(3):137-154

2. Cohen C, Clanet C (2016) Physics of ball sports. Europhys News 47(3):13-16

3. Achenbach E (1972) Experiments on the flow past spheres at very high Reynolds numbers. J Fluid Mech 54(3):565-575

4. Trackman (2014) Trackman Newsletter \#10. http://www.golfe rsadvantage.com/articles/TrackMan_Newsletter_2014.pdf

5. Nathan AM (2008) The effect of spin on the flight of a baseball. Am J Phys 76(2):119-124

6. Prandtl L (1953) Essentials of fluid dynamics. Hafner Publishing Company, New York, pp 345-366

7. Kensrud JR, Smith LV (2011) In situ lift measurement of sports balls. Procedia Eng 13:278-283

8. Kim J et al (2014) Inverse Magnus effect on a rotating sphere: when and why. J Fluid Mech 754:R2

9. Bearman P, Harvey J (1976) Golf ball aerodynamics. Aeronaut Q 27(2):112-122

10. Maccoll JW (1928) Aerodynamics of a spinning sphere. Aeronaut J 32(213):777-798

11. Briggs LJ (1959) Effect of spin and speed on the lateral deflection (curve) of a baseball; and the Magnus effect for smooth spheres. Am J Phys 27(8):589-596

12. Muto M, Tsubokura M, Oshima N (2012) Negative Magnus lift on a rotating sphere at around the critical Reynolds number. Phys Fluids 24(1):014102

13. Bush JWM (2013) The aerodynamics of the beautiful game. In: Clanet C (ed) Sports physics. Les Editions de l'Ecole Polytechnique, pp 171-192
14. Oggiano L, Sætran L (2010) Aerodynamics of modern soccer balls. Procedia Eng 2(2):2473-2479

15. Barber S, Chin S, Carré M (2009) Sports ball aerodynamics: a numerical study of the erratic motion of soccer balls. Comput Fluids 38(6): 1091-1100

16. Macha J et al (1991) Slotted-wall blockage corrections for disks and parachutes. J Aircr 28(9):592-597

17. Choi J, Jeon W-P, Choi H (2006) Mechanism of drag reduction by dimples on a sphere. Phys Fluids 18(4):041702

18. Cross R, Lindsey C (2014) Measurements of drag and lift on tennis balls in flight. Sports Eng 17(2):89-96

19. Lieberman BB et al (2001) Method for determining coefficients of lift and drag of a golf ball, Google Patents

20. Martin J, Smith LV, Kensrud JR (2012) Drag on sports balls using Doppler radar. Procedia Eng 34:134-139

21. Kensrud JR, Smith LV (2017) Drag and lift measurements of solid sports balls in still air. In: Proceedings of the institution of mechanical engineers, part $\mathrm{P}$ journal of sports engineering and technology, p 1754337117740749

22. Goff J, Smith W, Carré M (2011) Football boundary-layer separation via dust experiments. Sports Eng 14(2-4):139-146

23. Goff JE et al (2017) Creating drag and lift curves from soccer trajectories. Eur J Phys 38(4):044003

24. Kensrud J (2018) FLEX grip sports ball pitching machine tip, PCT/US2017/032887

25. Kensrud JR (2010) Determining aerodynamic properties of sports balls in situ, Washington State University

26. Lyu B et al (2018) Aerodynamics of Golf Balls in still air. In: Multidisciplinary digital publishing institute proceedings

Publisher's Note Springer Nature remains neutral with regard to jurisdictional claims in published maps and institutional affiliations. 